\title{
Optimal Control System of Automatic Navigation Aircraft Cluster for Mechanical Carrier Vehicles
}

\author{
Xu Ke \\ School of International Education, Wuhan University of Technology, Wuhan, China
}

Keywords: Vehicles; Control System; Automatic Navigation Aircraft; Domestic

\begin{abstract}
The fundamental difference among Steer by wire and other steering systems lies in that it cancelled the mechanical or hydraulic connecting device between the steering wheel and the turning wheel, thus it breaks through fixed transmission ratio limit of the meshing gear, and reduces the total clearance among the inertial, friction and transmission parts of the system, and finally improves the response speed and response accuracy of the system. Domestic research mainly focuses on algorithms related to Steer-by-Wire system, such as the algorithms of return torque and stability of the steering wheel. Domestic research doesn't form in-depth research of the communication protocol used in the system. And, FlexRay is through accurate and high speed communication network protocol to meet the requirements of SBW system when there are fault tolerance and time uncertainty of the message transmission, thus to improve the controllability, stability and safety of car handling. Therefore, the research of the SBW system's communication network is a research in a new field, and it is also crucial to improve the vehicle performance and gradually makes it more intelligent.
\end{abstract}

\section{Simulation and performance Comparison of the Software}

Assume that there are four nodes in a FlexRay network, and three of which are synchronized nodes. The cold boot process should be ignored while conduct the software simulation process. Besides, we should focus on the clock synchronization conditions of Cycle8 Cycle13--these six cycles, and each cycle should be simplified as ST segment and NIT segment. We should set the relevant protocol parameters meet the following equations gdCycle $=1000$, gdMacrotick $=1 \mu \mathrm{s}$, pMacroPerCycle=1000, gdStaticSlot=100MT.

In order to calculate the phase and frequency correction value of each node, we should write the FTM algorithm Matlab as: [offsetcorrection, ratecorrection]=FTM_algorithm (num, offsetarray, ownoffset). In the above equation, num is the number of synchronization nodes, for cSyncNodeMax=15, the maximum value of num of is 15; offset- array is the expected arrival time of certain synchronization frame, and it takes the node's local time as reference. Through FTM_algorithm function, we could obtain phase and frequency correction value of each node among Cycle10 Cycle11 as shown in table 1. In table 1, pdMicrotick is the length value of $\mu \mathrm{T}$, and its unit is $\mu \mathrm{s}$; and we take $\mu \mathrm{T}$ as unit of phase and frequency correction value.

Table 1 Phase and frequency correction value among Cycle10 Cycle11

\begin{tabular}{llll}
\hline \hline nodes & pdMicrotick $(\mu \mathrm{s})$ & $\begin{array}{l}\text { Phase correction } \\
\text { value }(\mu \mathrm{T})\end{array}$ & $\begin{array}{l}\text { Frequency } \\
\text { correction } \\
\text { value }(\mu \mathrm{T})\end{array}$ \\
\hline $\begin{array}{l}\text { Non synchronous } \\
\text { node }\end{array}$ & 0.1 & -820 & -43 \\
$\begin{array}{l}\text { synchronization } \\
\text { node 1 }\end{array}$ & 0.025 & 429 & 28 \\
$\begin{array}{l}\text { synchronization } \\
\text { node 2 }\end{array}$ & 0.025 & -29 & -3 \\
$\begin{array}{l}\text { synchronization } \\
\text { node 3 }\end{array}$ & 0.025 & -406 & -27 \\
\hline \hline
\end{tabular}




\section{The Real-time and Fault Tolerance Evaluation of Steering System of Flexray Wire}

\subsection{The FlexRay communication module hardware}

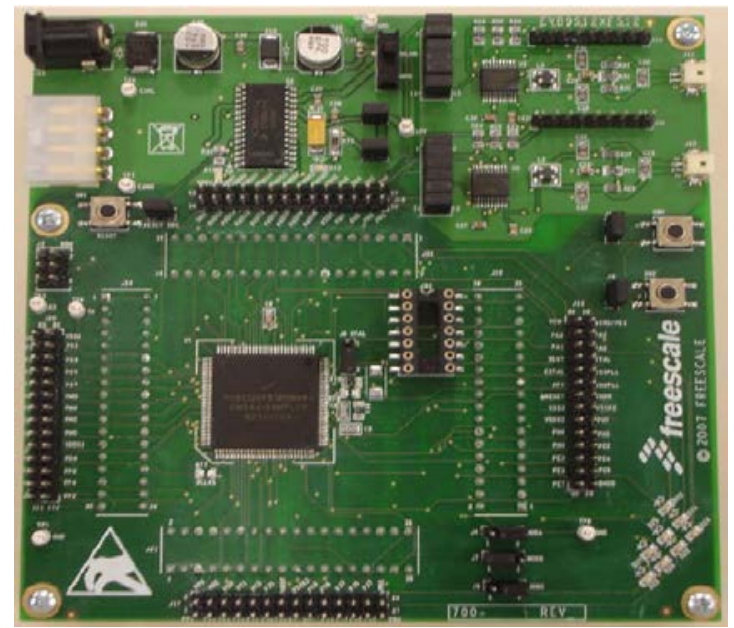

Fig. 1. EVB9S12XF512 evaluation board

In this paper, we choose the EVB9S12XF512 evaluation board of Freescale as basic ECU nodes, as shown in Figure1. EVB9S12XF512 includes a 16-bit-microcontroller: MC9S12XF512 with FlexRay communication controller embedded in it, two FlexRay bus drivers: TJA1080 and a high speed CAN bus driver: MC33742.

(1) Micro controller MC9S12XF512

There is a XGATE co processor, $512 \mathrm{~K}$ byte Flash and an embedded FlexRay communication controller in the micro controller MC9S12XF512. The package diagram of this micro controller is as shown in figure 2. In figure 2, XGATE is aimed to conduct fast interruption processing, thus it could reduce the load of CPU while conduct interrupt processing. Therefore, it provides a higher level of disruption, and through the sharing of some service procedures it could shorten the working time and process of CPU. XGATE, like CPU, is a programmable kernel that supports the C compiler. When the source is off, XGATE begins to run; when the interruption task is completed, it will stop all the clocks and wait for the next event, thus it could reduce power consumption. Therefore, although MC9S12XF512 is a 16 bit microcontroller, it can achieve the performance that of 32-bit microcontroller.

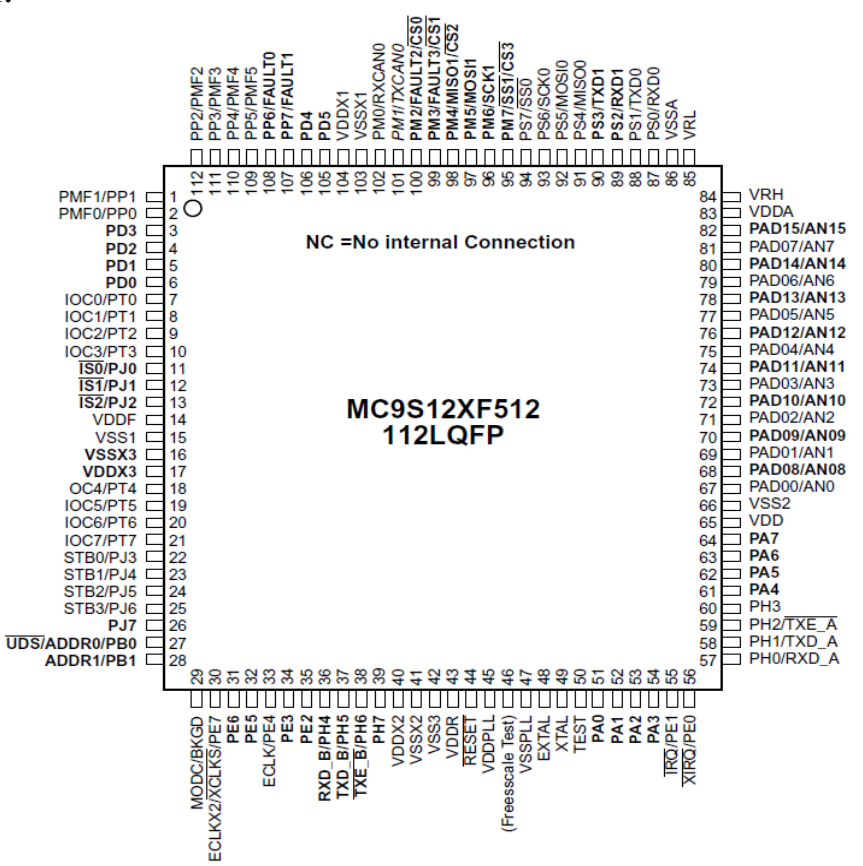

Fig. 2. The package diagram of the microcontroller MC9S12XF512 
(2) The FlexRay bus driver

The FlexRay bus driver uses TJA1080 produced by NXP company, and it could provide data rate up to $10 \mathrm{Mbps}$. So it is suitable for working during the $14 \mathrm{~V} 42 \mathrm{~V}$ voltage range. And the electromagnetic radiation of FlexRay bus driver is low, and the input differential mode is adopted in this bus driver. The IO terminal voltage of the bus driver can altered by itself accordingly, at the same time, there is BGE interface reserved for the bus monitor. The chip provides 4 kinds of bus states: low power state, idle state, data 1 state and data 0 state.

In order to achieve the voltage adaptive adjustment of the IO terminal, in the circuit, we connect drive voltage of the communication controller (namely the drive voltage of the microcontroller with the VIO pin of TJA1080. And then, no voltage conversion chip is needed to achieve the normal communication between TJA1080 and controller. The bus driver circuit that the channel B corresponds is similar to Figure 3, thus we left out this part.

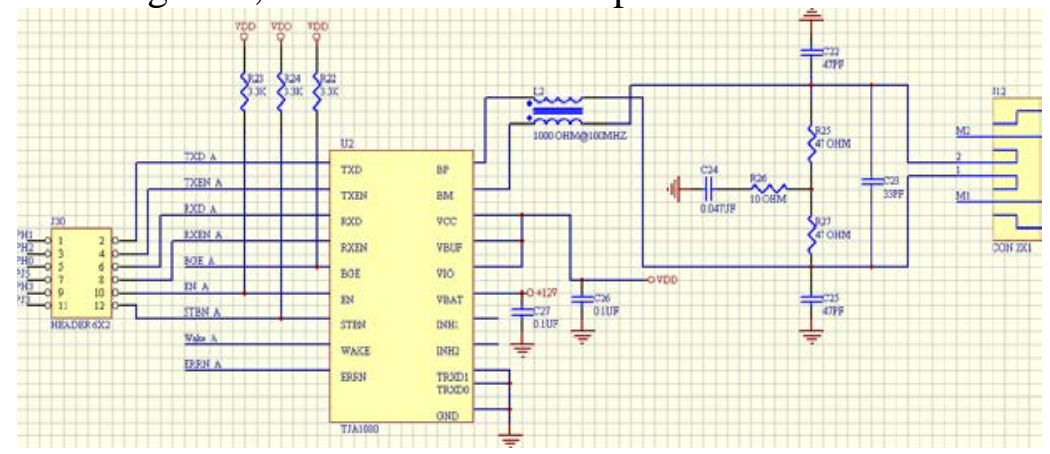

Fig.3. FlexRay bus driver circuit diagram

\subsection{Real Time and Fault Tolerance Evaluation}

We integrate the steering, yaw warning and electronic stability control system to get the message scheduling scheme shown in table 2-3. The ST segment transmits a ST message containing a variable steering drive ratio and a steering wheel torque, and at the same time it starts and synchronizes this message. Due to the fact that the message in DYN segment is triggered by the event, so when the sensors are in normal state, this segment only receives yaw warning and information related to steering in electronic stability control system; if a sensor failure happens, the correct variable steering transmission ratio or steering wheel aligning torque value will be sent in the previous dynamic slots (here the "correct" value could be obtained from the sending node based on the processing of the previous fault-tolerant calculation. Because that this aspect has nothing to do with FlexRay communication, not many researches are conducted) to correct the error value sent by ST segment in the cycle. In order to ensure the real-time performance of the system, in each cycle we should pre-use the fault-tolerance algorithm to calculate the variable steering drive ratio / steering wheel torque value as backup-information, when there is a fault in the sensor, the ST segment could directly send this value.

Table 2 Priority solution of DYN message (unit:MS)

\begin{tabular}{lllll}
\hline \hline$n$ & $j$ & $M d l m$ & $R t m$ & diffm \\
\hline DM1 & \multirow{2}{*}{2} & 355.5 & 118.5 & 237 \\
DM2 & & 361.5 & 120.5 & 241 \\
\hline \hline
\end{tabular}

$\operatorname{diff}_{\mathrm{Dm} 2} \geq \operatorname{diff} f_{\mathrm{Dm} 1}$ indicates that the worst-case response time of DM1 is close to the deadline. In order to guarantee the schedulability of the system, we should first send DM1, and assign the smaller frame ID value to FrameIDDM1. Thus we can get the time slot arrangement in table 3, namely the corresponding dynamic DM1 timeslot 1 (namely slot67), and the corresponding dynamic DM2 2 time slot (namely slot68). And then we receive information related to steering of the yaw warning and electronic stability control systems in the dynamic slot 3 and slot 4 (i.e. slot69, slot70). Due to the fact that the message sending timing is associated with the scheduling of the node, and the two nodes belong not to wire-control steering network, so we don't compare the 
arrangement orders while receiving time slots.

Table 3 Time triggered schedule

\begin{tabular}{|c|c|c|c|}
\hline Time slot & MB delivery & MB receive & FIFO receive \\
\hline \multicolumn{4}{|c|}{ STsegment } \\
\hline slot1 & $\begin{array}{l}\text { n1,chAB,SM1(start and synchronous frame, and the } \\
\text { load data is the variable steering ratio) }\end{array}$ & $\mathrm{n} 2, \mathrm{chAB}$ & \\
\hline slot4 & $\begin{array}{l}\text { n2,chAB,SM2(start and synchronous frame, and the } \\
\text { load data is the steering wheel torque) }\end{array}$ & $\mathrm{n} 1, \mathrm{ch} A \mathrm{~B}$ & \\
\hline \multicolumn{4}{|c|}{ DYN segment } \\
\hline slot67 & n1,chA,DM1(the adjusted variable steering ratio) & & n2,chA \\
\hline slot68 & n2,chA,DM2(the adjusted steering wheel torque) & & $\mathrm{n} 1, \mathrm{chA}$ \\
\hline slot69 & n3,chA,DM3(information of yaw warning system) & & $\mathrm{n} 1$ \& n2, chA \\
\hline slot70 & $\begin{array}{l}\text { n4,chA,DM4(information of electronic stability } \\
\text { control system) }\end{array}$ & & $\mathrm{n} 1$ \& n2,chA \\
\hline
\end{tabular}

In table 3, the message sending of ST segment is achieved according to the schedule table, while DYN segment obtains the present message scheduling by dynamic programming algorithm. Therefore, we can adjust the arrangement according to actual condition. In this table, SM stands for the Static message, namely ST message; The abbreviation of Dynamic message is DM, namely DYN message; chAB means sending or receiving data with double channels, and chA means sending or receiving data only with channel A; $\mathrm{n} 3$ and $\mathrm{n} 4$ are respectively the nodes in yaw warning system and electronic stability control system. Due to the fact that the microcontroller MC9S12XF512 does not support the dual channel communication of DYN segment, so we only use channel A to send and receive message.

\section{Conclusion}

In the aspect of clock synchronization, we introduce the concept of phase deviation state diagram. We replace the real time intervals with continuous time intervals, and based on the more accurate clock discrepancy information to improve the network synchronization accuracy; and according to the weight changes of the state diagram, and conduct real-time and dynamic evaluation of the synchronization clock performance of the synchronization nodes and the stability of the network to make each node's local time be closer to the global time.

\section{References}

[1] ZHANG, CHEN, XI. System dynamic modelling and adaptive optimal control for automatic clutch engagement of vehicles[J]. Proceedings of the Institution of Mechanical Engineers -- Part D, 2002, 216(12):983-991.

[2] Zhen Z, Zhang Z, Zhang J. Guidance and Control Techniques of Carrier-Based Aircraft for Automatic Carrier Landing[J]. Transactions of Nanjing University of Aeronautics \& Astronautics, 2017, 34(6):600-608.

[3] Zhao X, Chen H. Optimal control of the automatic steering system[C]// IEEE International Conference on Vehicular Electronics \& Safety. 2011.

[4] Boskovic J, Redding J. An Autonomous Carrier Landing System for Unmannned Aerial Vehicles[C]// Aiaa Guidance, Navigation, \& Control Conference. 2009.

[5] Dou R, Duan H. Lévy flight based pigeon-inspired optimization for control parameters optimization in automatic carrier landing system [J]. Aerospace Science \& Technology, 2017, 61:11-20.

[6] O'Connor M L, Elkaim G H, Parkinson B W. Carrier-Phase DGPS for Closed-Loop Control of Farm and Construction Vehicles [J]. Navigation, 1996, 43(2):166-178. 